\title{
Stroke - shoulder pain and upper limb function
}

\begin{abstract}
Introduction: Stroke causes disability and pain, especially shoulder pain. Often, shoulder pain, has a not completely known mechanism and evolution. ${ }^{1,2}$
\end{abstract}

Objectives: Assess shoulder pain and its impact in upper limb function, in patients who had suffered a stroke within 6 months.

Methods: Observational study. Included patients at discharge from an inpatient rehabilitation centre, from November 2019 until February 2020. Assessment was done using validated Portuguese versions of the Brief Pain Inventory (BPI) and Fugl Meyer Assessment Scale (FMAS)

Results: Of 32 patients screened, 26 fulfilled the inclusion criteria, 7 were females (26 ,9\%), with mean age of $60,7 \pm 10$ years. Mean values of BPI Severity and Interference were $3,2 \pm 1,6$ and $2,4 \pm 1,8$, respectively. The mean values of the FMAS Motor Function and Passive Articular Movement were 38,8 $\pm 23,2$ and 20,3 $\pm 2,3$, respectively. Analysing the association between both subscales of BPI and both Subscales of FMAS negative correlations were found to be statistically significant with a confidence interval of $95 \%$ but no statistically significant correlation was found between BPI Severity and FMAS motor function. Six patients $(23 \%)$ received a local injection for shoulder pain. Analysing both groups, BPI Severity and both subscales of FMAS showed a statistically significant difference (p-values of 0,0083, 0,0031 and 0,0056, respectively) for a Wilcoxon/KruskalWallis test with a confidence interval of $95 \%$

Discussion/Conclusions: Patients with voluntary upper limb movements after a stroke tend to have less shoulder pain. Local injection was an effective intervention for shoulder pain. The major limitation of this study is the small sample size.
Volume 7 Issue I - 2022

\author{
Carvalho Sá P,' Ferro I,' Pires C,' Pascoal \\ A,' Lourenço A,' Constantino J,' Martins L,' \\ Campos I,' Valério M, ${ }^{2}$ Mendes B, ${ }^{3}$ Reis M S, ${ }^{4}$ \\ Laíns J.,5 \\ 'Centro de Medicina de Reabilitação da Região Centro - \\ Rovisco Pais, Portugal \\ ${ }^{2}$ Centro Hospitalar do Médio Tejo, Serviço de Pneumologia, \\ Portugal \\ ${ }^{3}$ Centro Hospitalar do Baixo Vouga, Serviço de Medicina Física e \\ de Reabilitação, Portugal \\ ${ }^{4}$ Universidade de Coimbra, CIEPQPF, Departamento de \\ Engenharia Química, Portugal \\ ${ }^{5}$ Faculdade de Medicina, Universidade de Coimbra, Portugal
}

Correspondence: Pedro Carvalho Sá, Centro de Medicina de Reabilitação da Região Centro, Rovisco Pais, Quinta da Fonte Quente, Tocha, Cantanhede, Portugal, 3064-908,

Tel 00351912378922, Email pedro.sa.7@hotmail.com

Received: December 28, 202I | Published: January 06, 2022
Abbreviations: BPI, Brief Pain Inventory; FMAS, Fugl Meyer Assessment Scale; INE, National Institute of Statistics; SD, standard deviation

\section{Introduction}

Cerebrovascular disease is a major cause of death and it is the leading cause of disability in adults - in 2013, there were globally 6.5 million deaths from stroke and 113 million DALYs due to stroke. ${ }^{1}$ In Portugal, data from the National Institute of Statistics (INE) revealed that cardiovascular diseases were the major cause of death $(29.8 \%)$ in 2015 with stroke being the most frequent cause of death within this group of diseases.

Stroke can also be a cause of pain that can occur through a variety of mechanisms - central post-stroke pain, complex regional pain syndrome, and pain associated with spasticity, among other shoulder pathologies and dysfunctions. ${ }^{2}$ Shoulder pain is one of the common symptoms related to stroke and it can be associated with various mechanisms and have different clinical courses from one patient to another. ${ }^{2-4}$

Pain is a limiting aspect of functionality and has a major negative impact on the quality of life and in multiple areas of functioning such as cognition, emotion, and behavior. ${ }^{5,6}$

The Brief Pain Inventory (BPI) is a self-administered questionnaire for chronic pain conditions. It is available in a short (nine items) and long (17 items) form. The BPI short form is more frequently used. The questionnaire is composed of pain drawing diagrams about pain severity, pain relief treatment or medication, and pain interference with multiple aspects of the patient's life.?

One of the most widely recognized and clinically relevant measures of body function impairment after stroke is the Fugl-Meyer
Assessment Scale (FMAS). Of its 5 domains (motor, sensory, balance, range of motion, joint pain), the motor domain has well-established reliability and validity as an indicator of motor impairment severity across different stroke recovery time points. ${ }^{8}$

This study focuses on shoulder pain and its impact in the upper limb function, in patients who recently suffered a stroke (within 6 months of the event).

\section{Methods}

\section{Study design and data collection}

This cross-sectional observational study was performed at an inpatient rehabilitation center from November 2019 till February of 2020. This study was conducted according to STROBE guidelines.

The inclusion criteria of the study were as follows:

a) First stroke

b) Hemiplegic due to a stroke less than 6 months ago

c) Ability to comprehend and answer in Portuguese

d) Cognitive capacity (Mini Mental State Examination>19)

e) Shoulder pain at the hemiplegic side (at rest or with mobilization)

f) Shoulder pain that began after stroke.

The following exclusion criteria were applied:

a) Hemiplegic due to a stroke more than 6 months ago

b) Chronic generalized pain previous to the stroke

c) Cognitive impairment (Mini Mental State Examination < 19) 
d) Other debilitating diseases (Parkinson's disease, polineuropathy, chronic cardiac, renal or liver disease cancer, or limb amputation)

e) Previous shoulder pathology

f) Complex regional limb pain (hand-shoulder syndrome)

g) Decompensated psychiatric disorder.

This protocol was approved by the competent human research ethics committee, from our Rehabilitation Center, and every patient provided informed consent before being enrolled in the study.

For each patient demographic data was collected as well as treatment strategies that were performed in each case. The validated Portuguese versions of the Brief Pain Inventory and of the Fugl Meyer Assessment Scale (upper limb) were used to access both pain and sensorimotor impairment.

\section{Statistical analysis}

We summarized the characteristics of study populations using descriptive statistical methods with frequencies and percentage, mean, and standard deviation (SD).

The correlation between the two questionnaires was determined with Spearman correlation with $95 \%$ confidence interval. To evaluate the effectiveness of a specific treatment approach the Wilcoxon/ Kruskal-Wallis test was used with a confidence interval of $95 \%$.

All calculations were performed using JMP PRO ${ }^{\circledR}$ version 15, SAS Institute Inc.

\section{Outcome measures}

The primary goal was to determine whether treatment strategies for hemiplegic shoulder pain were effective. For that we ascertained if there was a statistically significant difference on the grading of the scales used in this study between both groups (the one taking and the other not taking pain medication). Another goal was to establish whether shoulder pain and upper limb function were correlated. This was done by correlating both scales used and see if there was statistical significance between them.

\section{Results}

From a total of 32 patients discharged from our Rehabilitation Center in the referred period, 26 fulfilled the inclusion criteria, being that 19 patients were males $(73.1 \%)$, with a mean age of $60.7 \pm 10$ years. Thirteen $(50 \%)$ patients presented with a left hemispheric stroke and $13(50 \%)$ with a right hemispheric stroke. An ischemic stroke was observed in 16 patients $(61.5 \%)$ and 10 patients $(38.5 \%)$ had a hemorrhagic stroke. The average time until the onset of shoulder pain was $6 \pm 2.5$ weeks $(\min =2$; $\max =12)$; the average time from the beginning of pain complaints till the study was conducted was $10 \pm 4.2$ weeks $(\min =4 ; \max =22)$. Demographic and disease related data are presented in (Table 1).

The therapeutic strategies performed are displayed in. (Table 2) Twenty-two patients $(84.6 \%)$ were submitted to physical modalities during physical therapy and 18 patients $(69.2 \%)$ were included in hydrokinesitherapy during treatment. Eleven patients $(42.3 \%)$ were given pain medication whereas only 6 patients $(23 \%)$ received a local injection of an anesthetic or a corticosteroid to ameliorate the pain. Due to a reduced number of patients in each group, both intra-articular and soft tissue injections were grouped.
Table I Demographic and disease related data

\begin{tabular}{|c|c|c|c|}
\hline Characteristics & $\mathbf{n}$ & $\%$ & Mean ( \pm SD) \\
\hline Age (years) & & & $60.7 \pm 10$ \\
\hline Sex (male) & 19 & 73.1 & \\
\hline Left hemispheric stroke & 13 & 50 & \\
\hline Right hemispheric stroke & 13 & 50 & \\
\hline Ischemic stroke & 16 & 61.5 & \\
\hline $\begin{array}{l}\text { - Lacunar anterior circulation infarct } \\
(\mathrm{LACl})\end{array}$ & 3 & II.5 & \\
\hline $\begin{array}{l}\text { - Parcial anterior circulation infarct } \\
\text { (PACI) }\end{array}$ & 8 & 30.8 & \\
\hline $\begin{array}{l}\text { - Total anterior circulation infarct } \\
\text { (TACl) }\end{array}$ & 2 & 7.7 & \\
\hline \multirow[t]{2}{*}{ - Posterior circulation infarct (POCI) } & 2 & 7.7 & \\
\hline & I & 3.8 & \\
\hline Hemorrhagic stroke & 10 & 38.5 & \\
\hline Onset of pain after stroke (weeks) & & & $6 \pm 2.5$ \\
\hline $\begin{array}{l}\text { Onset of paint until evaluation in the } \\
\text { study (weeks) }\end{array}$ & & & $10 \pm 4.2$ \\
\hline
\end{tabular}

Table 2 Therapeutic interventions

\begin{tabular}{lll}
\hline Therapy & $\mathbf{n}$ & $\%$ \\
\hline Physical modalities & 22 & 84.6 \\
Hydrokinesiotherapy & 18 & 69.2 \\
Pain medication & $1 \mathrm{I}$ & 42.3 \\
Local injection & 6 & 23.1 \\
Assistive devices & 6 & 23.1 \\
Botulinum toxin & 4 & 15.4 \\
Oral antispasticity medication & 3 & $1 \mathrm{I} .5$ \\
Supraescapular nerve block & 3 & 11.5 \\
\hline
\end{tabular}

The mean value of the BPI domains was $3.2 \pm 1.6$ for BPI Severity and $2.4 \pm 1.8$ for BPI Interference. Also, for the FMAS domains, mean FMAS Motor Function was $38.8 \pm 23.3$ and mean FMAS Passive Articular Movement was 20.3 \pm 2.3 .

Analyzing the association between both subscales of BPI (subscales Severity and Interference) and both Motor Function and Passive Articular Movement (subscales of FMAS) negative correlations were found to be statistically significant at all correlations tested with a confidence interval of $95 \%$. The exception was the BPI Severity and FMAS passive articular movement, where no statistically significant correlation was found (Table 3).

Treatment approaches and its effects on the domains of the scales used are represented in Table 4 and Table 5. BPI Interference, FMAS Motor Function and Passive Articular Movement differ in a statistical significant way upon the utilization of an injection ( $\mathrm{p}$ values of $0,0083,0,0031$ and 0,0056 ) for a Wilcoxon/Kruskal-Wallis test with a confidence interval of $95 \%$. The other interventions did not show statistically significant differences in the domains of the scales used. 
Table 3 Spearman correlation between domains of the BPI and FMAS

\begin{tabular}{lll}
\hline Scale domain & Spearman $\rho$ & p-value \\
\hline $\begin{array}{l}\text { BPI Severity and FMAS Motor } \\
\text { Function }\end{array}$ & -0.3273 & 0.1025 \\
$\begin{array}{l}\text { BPI Severity and FMAS Passive } \\
\text { Articular Movement }\end{array}$ & $-0.448 \mathrm{I}$ & 0.0217 \\
$\begin{array}{l}\text { BPI Interference and FMAS Motor } \\
\text { Function }\end{array}$ & -0.408 & 0.0386 \\
$\begin{array}{l}\text { BPI Interference and FMAS Passive } \\
\text { Articular Movement }\end{array}$ & -0.4495 & 0.0212 \\
\hline
\end{tabular}

Table 4 Therapeutic interventions and Brief Pain Inventory

\begin{tabular}{ll} 
BPI domains and therapeutic interventions & p-value \\
\hline BPI Severity with physical modalities & 0.163 \\
BPI Severity with hydrokinesiotherapy & 0.7522 \\
BPI Severity with pain medication & 0.0912 \\
BPI Severity with local injection & 0.0083 \\
BPI Interference with physical modalities & 0.2635 \\
BPI Interference with hydrokinesiotherapy & 0.2148 \\
BPI Interference with pain medication & 0.1792 \\
BPI Interference with local injection & 0.1152
\end{tabular}

Table 5 Therapeutic interventions and Fugl Meyer Assessment Scale

\begin{tabular}{ll}
\hline FMAS domains and therapeutic interventions & p-value \\
\hline FMAS Motor Function with physical modalities & 0.1884 \\
FMAS Motor Function with hydrokinesiotherapy & 0.3738 \\
FMAS Motor Function with pain medication & 0.9586 \\
FMAS Motor Function with local injection & 0.003 I \\
FMAS Passive Articular Movement with physical modalities & 0.0167 \\
FMAS Passive Articular Movement with hydrokinesiotherapy & 0.822 \\
FMAS Passive Articular Movement with pain medication & 0.189 \\
FMAS Passive Articular Movement with local injection & 0.0056
\end{tabular}

\section{Discussion}

After a stroke, both active and passive upper limb movement (evaluated through both subscales of FMAS, respectively) were associated with less pain (subscales of BPI); no evaluated pain intervention was in fact effective, except for a local therapeutic injection.

The use of pain medication showed a positive tendency $(p=0,0912)$ - possibly, with a larger sample of patients a statistically significant difference could be shown. It is important to highlight, that only 11 (out of the 26) patients were actually taking pain medication. The others stated that the pain was only present during physiotherapy sessions, and that they did not want to take any medication, even when it was offered.

As ascertained previously, both subscales of the BPI were negatively associated with Motor Function and Passive Articular Movement (subscales of FMAS). So, shoulder pain after stroke is, as showed in this study, of relevance to upper limb movement. Interventions used for its treatment are largely lacking solid evidence.
The precise reasons for these results are still unknown. Regardless, some speculation can be made. As previously stated, mechanisms of pain after a stroke seem to be multiple in different patients and maybe more than one present in each patient. ${ }^{2}$ Understanding the most likely cause of shoulder pain in each patient might enlighten clinicians as to which specific treatment can be more effective.

A recent systematic review for interventions in post-stroke shoulder pain showed positive results for acupuncture, orthoses, botulinum toxin injection, electrical stimulation and aromatherapy. ${ }^{9}$ However, findings were often inconsistent across outcome measures or follow-up times, reinforcing the lack of evidence and need for individualization and standardization of approaches.

Shoulder pain is relevant as a symptom, but also in a functional level. In a previous study, post-stroke patients with shoulder pain had reduced upper limb function and lower daily functional independence (evaluated with the Functional Independence Measure) when compared to patients without shoulder pain. ${ }^{10}$ In addition, post-stroke patients with impaired quality of life are likely to be functionally dependent. ${ }^{11}$ Therefore, managing pain is important, not only for its relief, but also to improve upper limb function, functional independence, and ultimately, for their quality of life.

The most relevant limitations of this study are its cross-sectional design (only one evaluation was conducted) and the small sample of patients included. Also, some interventions were grouped and these groups may be heterogenous. Evaluating specific approaches within each group - for instance, certain types of pain medication or physical modalities - could lead to different results. In spite of this, relevant data was obtained.

Prospective longitudinal studies, beginning in the acute phase after stroke, and maintaining the assessment of patients in the sub-acute period, are necessary for a better knowledge both of the evolution and possible early interventions in shoulder pain after a stroke.

\section{Conclusion}

After a stroke both active and passive upcper limb range of movement was associated with less pain. Nevertheless, none of the evaluated pain intervention evaluated was effective except for a local injection. Prospective studies are necessary for a better knowledge both of the evolution and possible interventions in shoulder pain after stroke.

\section{Statement of ethics}

This study was conducted according the World Medical Association Declaration of Helsinki.

The study protocol was approved by our Rehabilitation ethics committee (CMRRC - Rovisco Pais).

Written informed consent was obtained from participants (legal guardian/next of kin) to participate in the study.

\section{Acknowledgments}

None.

\section{Conflicts of interest}

The authors have no conflicts of interest to declare.

\section{Funding}

No sponsor or funding was required to develop this study. 


\section{Author contributions}

All authors contributed to the development of this article according to the ICMJE Criteria for Authorship.

\section{Data availability statement}

All data generated or analyzed during this study are included in this article and its supplementary material files. Further enquiries can be directed to the corresponding author.

\section{References}

1. Feigin VL, Krishnamurthi RV, Parmar P, et al. Update on the global burden of ischemic and hemorrhagic stroke in 1990-2013: the GBD 2013 study. Neuroepidemiology. 2015;45:161-176.

2. Treister AK, Hatch MN, Cramer SC, et al. Demystifying Poststroke Pain: From Etiology to Treatment. PMR. 2017;9(1):63-75.

3. Lydia Martín-Martín et al; Association between physiological and subjective aspects of pain and disability in post-stroke patients with shoulder pain: a cross-sectional study. J Clin Med. 2019;8(8):1093.

4. Gaitan M, Bainbridge M, Parkinson S, et al. Characteristics of the shoulder in patients following acute stroke: a case series. Top Stroke Rehabil. 2019;26(4):318-325.
5. Lemos BO, Cunha AM, Cesarino CB, et al. The impact of chronic pain on functionality and quality of life of the elderly. BrJP 2019;2(3):237-241.

6. Turk DC, Fillingim RB, Ohrbach R, et al. Assessment of psychosocial and funcional impact of chronic pain. The Journal of Pain. 2016;17(9):T21T49.

7. Poqueta N, Lin C. Brief pain inventory (BPI). Journal of Physiotherapy. 2016;62:52.

8. Sullivan KJ, Tilson JK, Cen SY, et al. Fugl-meyer assessment of sensorimotor function after stroke - standardized training procedure for clinical practice and clinical trials. Stroke. 2011;42:427-432.

9. Dyer S, Mordaunt DA, Adey-Wakeling Z. Interventions for post-stroke shoulder pain: an overview of systematic reviews. International Journal of General Medicine. 2020;13:1411-1426.

10. Nickel R, Lange M, Stoffel DP, et al. Upper limb function and functional independence in patients with shoulder pain after stroke. Arq Neuropsiquiatr 2017;75(2):103-106.

11. Raju RS, Sarma PS, Pandian JD. Psychosocial problems, quality of life, and functional independence among Indian stroke survivors. Stroke. 2010;41(12):2932-2937. 